Pedro Ángel Sangucho-Verdezoto; Ana Abigail López-Guerrero; María Margarita Escobar-Martínez; Jenny Irene Plasencia-Criollo

http://dx.doi.org/10.35381/s.v.v4i8.1009

\title{
Enfoque médico integral de la enfermedad trofoblástica gestacional: Reporte de un Caso Clínico
}

\section{A comprehensive medical approach to gestational trophoblastic disease: A Clinical Case Report}

\author{
Pedro Ángel Sangucho-Verdezoto \\ psangucho9720@uta.edu.ec \\ Universidad Técnica de Ambato, Ambato \\ Ecuador \\ https://orcid.org/0000-0003-0255-8609 \\ Ana Abigail López-Guerrero \\ anbilo18@hotmail.com \\ Universidad Técnica de Ambato, Ambato \\ Ecuador \\ https://orcid.org/0000-0002-3565-4296 \\ María Margarita Escobar-Martínez \\ magy.e87@hotmail.com \\ Universidad Técnica de Ambato, Ambato \\ Ecuador \\ https://orcid.org/0000-0002-8352-4090 \\ Jenny Irene Plasencia-Criollo \\ ienny.plasencia@hotmail.com \\ Universidad Técnica de Ambato, Ambato \\ Ecuador \\ https://orcid.org/0000-0003-1377-4964
}

Recepción: 15 de junio 2020

Revisado: 28 de julio 2020

Aprobación: 25 de agosto 2020

Publicación: 03 de septiembre 2020 
Pedro Ángel Sangucho-Verdezoto; Ana Abigail López-Guerrero; María Margarita Escobar-Martínez; Jenny Irene Plasencia-Criollo

\title{
RESUMEN
}

La práctica médica diaria, siempre debe ser ejecutada de una manera integral en todos los niveles de atención del Sistema Nacional de Salud. Objetivo: Análisis de caso de neoplasia trofoblástica gestacional, dándole un enfoque integral desde la visión de un Especialista en Medicina Familiar. Metodología: Presentación de caso clínico, observacional descriptivo. Resultados: La enfermedad trofoblástica gestacional es una patología poco frecuente, un pequeño porcentaje de los casos diagnosticados (2\%), pueden convertirse desde el punto de vista clínico e histopatológico en una enfermedad maligna, el coriocarcinoma con mayor frecuencia. Conclusiones: Este caso clínico trata una neoplasia trofoblástica gestacional, que desarrolló en una mujer adolescente, primigesta, sin antecedentes patológicos personales, ni familiares de importancia. Sin embargo, existen consideraciones sociales, familiares y culturales que también deben ser tomadas en cuenta para el manejo hospitalario.

Descriptores: Neoplasias uterinas; medicina familiar y comunitaria; salud pública. (Fuente DeCS, 2020).

\begin{abstract}
Daily medical practice must always be carried out in a comprehensive manner at all levels of care of the National Health System. Objective: Analysis of a case of gestational trophoblastic neoplasia, giving it a comprehensive approach from the perspective of a Family Medicine Specialist. Methodology: Presentation of a clinical case, descriptive observational. Results: Gestational trophoblastic disease is an uncommon pathology, a small percentage of diagnosed cases (2\%), can become a malignant disease from a clinical and histopathological point of view, choriocarcinoma more frequently. Conclusions: This clinical case treats a gestational trophoblastic neoplasia, which developed in an adolescent woman, primigravida, with no personal or family pathological history of importance. However, there are social, family and cultural considerations that must also be taken into account for hospital management.
\end{abstract}

Descriptors: Uterine neoplasms; family practice; public health. (Source DeCS, 2020). 
Revista Arbitrada Interdisciplinaria de Ciencias de la Salud. SALUD Y VIDA

Volumen 4. Número 8. Año 4. Julio - Diciembre 2020

Hecho el depósito de Ley: FA2016000010

ISSN: 2610-8038

FUNDACIÓN KOINONIA (F.K)

Santa Ana de Coro, Venezuela.

Pedro Ángel Sangucho-Verdezoto; Ana Abigail López-Guerrero; María Margarita Escobar-Martínez; Jenny Irene Plasencia-Criollo

\section{INTRODUCCIÓN}

La enfermedad trofoblástica gestacional se produce por una proliferación anormal de tejido coriónico a nivel de la cavidad uterina, se produce un aumento en la secreción de la fracción beta de la HCG (gonadotrofina coriónica humana). Por lo tanto, la determinación de los niveles séricos de esta sustancia se constituye en un parámetro importante para establecer el diagnóstico de esta patología 123.

Los factores de riesgo involucrados en el desarrollo de esta enfermedad son múltiples, entre ellos se encuentran: menores de 20 años y mayores de 40 años, antecedentes de abortos a repetición y embarazos molares previos, el déficit en la dieta diaria del consumo de betacarotenos, el grupo sanguíneo $\mathrm{A}, \mathrm{AB}$ y el factor $\mathrm{RH}$ negativo ${ }^{4}$.

\section{Presentación del caso}

El presente caso se trata de una mujer de 19 años de edad, soltera, nacida y residente en el cantón la Maná, provincia de Cotopaxi, con un nivel de instrucción secundaria incompleta, sin antecedentes patológicos personales ni familiares de importancia, como antecedentes gineco-obstétricos: menarquia a los 13 años, ciclos menstruales regulares, fecha de última menstruación de 15/07/2018, con una amenorrea de 12,3 semanas cuando acude en busca de atención médica, un inicio de vida sexual activa a los 16 años, con 3 parejas sexuales, una planificación familiar ocasional con método de barrera, no embarazo ni abortos previos.

La paciente acude al centro de salud de la localidad con un cuadro clínico caracterizado por sangrado vaginal en moderada cantidad, más un dolor abdominal continuo e intenso a nivel de hipogastrio y mesogastrio, adicionalmente la paciente refiere que "su vientre ha crecido mucho en las últimas semanas".

Con estos antecedentes luego de ser valorada en la unidad de salud, le realizaron una ecografía pélvica y transvaginal, dando como resultado la visualización de imágenes sugestivas a una tormenta de nieve, acompañado de un aumento del diámetro del anexo 
Pedro Ángel Sangucho-Verdezoto; Ana Abigail López-Guerrero; María Margarita Escobar-Martínez; Jenny Irene Plasencia-Criollo

derecho. Se establece de esta forma el diagnóstico inicial de un embarazo molar por lo que decide realizar la transferencia hacia el Hospital Provincial General de Latacunga.

En esa casa de salud al momento del ingreso en el área de emergencia se constataron varias alteraciones en las cifras de signos vitales (tabla 1).

Por la medición de tensiones arteriales altas, se inicia un protocolo de preeclampsia, según lo que indica la guía de práctica clínica del Ministerio de Salud Pública ${ }^{5}$. Se recibe a una paciente estable, con funciones mentales superiores conservadas, los hallazgos al examen físico fueron palidez generalizada, diaforesis, altura de fondo uterino de $22,5 \mathrm{~cm}$ y un sangrado vaginal achocolatado, sin mal olor, en escasa cantidad, de aproximadamente 34 horas de evolución, desde que iniciaron las manifestaciones clínicas.

Tabla 1. Signos vitales, nivel de conciencia y parámetros antropométricos.

\begin{tabular}{|l|l|}
\hline \multicolumn{1}{|c|}{ MEDICIÓN } & \multicolumn{1}{c|}{ VALOR } \\
\hline Tensión arterial & $160 / 100$ \\
\hline Frecuencia cardíaca & 108 \\
\hline Frecuencia respiratoria & 20 \\
\hline Temperatura & $38,5^{\circ} \mathrm{C}$ \\
\hline Saturación de oxígeno & $92 \%$ \\
\hline Estado de conciencia & alerta \\
\hline Peso & $54 \mathrm{~kg}$ \\
\hline Talla & $163 \mathrm{~cm}$ \\
\hline Índice de masa corporal & 20,3 \\
\hline
\end{tabular}

Fuente: Archivos clínicos. Elaborado por: Autores. 
Pedro Ángel Sangucho-Verdezoto; Ana Abigail López-Guerrero; María Margarita Escobar-Martínez; Jenny Irene Plasencia-Criollo

Los estudios complementarios solicitados a nivel sanguíneo demostraron varias alteraciones (tabla 2). Entre las que se destacan una disminución de los niveles séricos de hemoglobina y plaquetas; con un aumento de las cifras sanguíneas de fracción beta de gonadotropina coriónica humana (BHCG). Además, hubo otros hallazgos en las pruebas de sangre que son característicos por representar un factor de riesgo para el desarrollo de esta patología (tabla 3). A nivel ecográfico se observó quistes tecaluteínicos en ovario derecho más imágenes en tormenta de nieve (figura 1 y 2 ).

Tabla 2. Hallazgos - pruebas de laboratorio.

\begin{tabular}{|l|c|}
\hline \multicolumn{1}{|c|}{ PRUEBA } & VALOR \\
\hline Hemoglobina & 8,1 \\
\hline Plaquetas & 80.000 \\
\hline BHCG (fracción beta de gonadotrofina coriónica humana) & 500.000 \\
\hline
\end{tabular}

Fuente: Archivos clínicos.

Elaborado por: Autores.

\section{Tabla 3: Otros resultados de laboratorio}

\begin{tabular}{|l|l|}
\hline \multicolumn{1}{|c|}{ PRUEBA } & RESULTADO \\
\hline Grupo sanguíneo & AB \\
\hline Factor $\mathrm{RH}$ & Negativo \\
\hline
\end{tabular}

Fuente: Archivos clínicos.

Elaborado por: Autores. 
Pedro Ángel Sangucho-Verdezoto; Ana Abigail López-Guerrero; María Margarita Escobar-Martínez; Jenny Irene Plasencia-Criollo

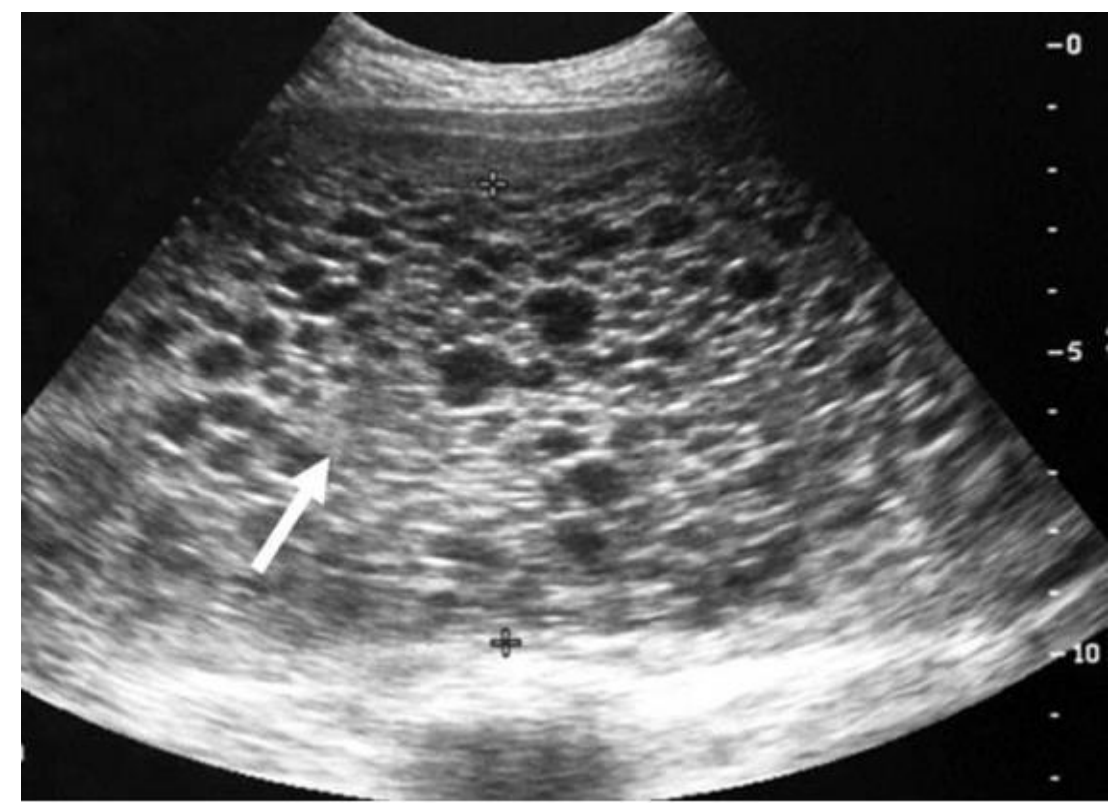

Figura 1: Imagen en tormenta de nieve característica de una enfermedad trofoblástica. Fuente: Historia clínica.

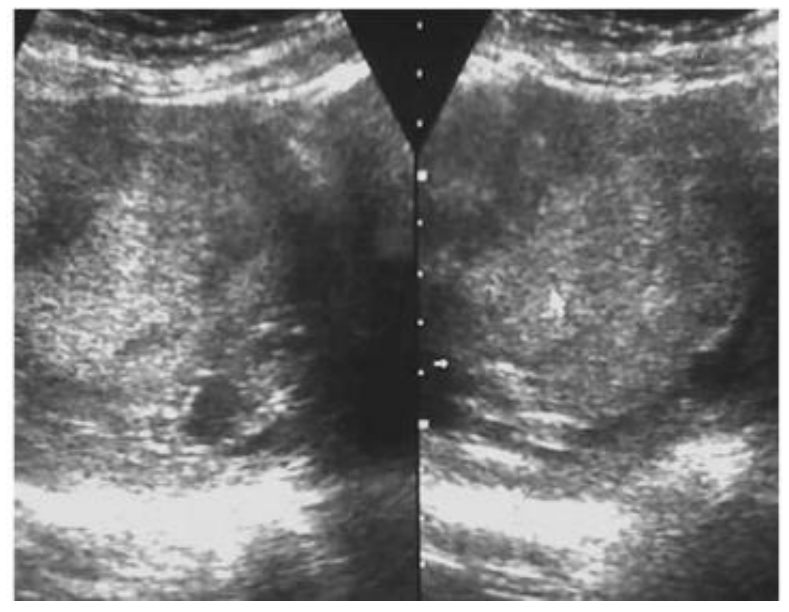

Figura 2: Imágenes ecolúcidas de aspecto quístico a nivel de ovario derecho. Fuente: Historia clínica. 
Revista Arbitrada Interdisciplinaria de Ciencias de la Salud. SALUD Y VIDA

Volumen 4. Número 8. Año 4. Julio - Diciembre 2020

Hecho el depósito de Ley: FA2016000010

ISSN: 2610-8038

FUNDACIÓN KOINONIA (F.K)

Santa Ana de Coro, Venezuela.

Pedro Ángel Sangucho-Verdezoto; Ana Abigail López-Guerrero; María Margarita Escobar-Martínez; Jenny Irene Plasencia-Criollo

El siguiente paso fue la realización de una AMEU (aspiración manual endouterina), en la cual se extrajo gran cantidad de tejido coriónico, con una pérdida de aproximadamente de $1800 \mathrm{cc}$ de sangre, por lo que la paciente tuvo que ser trasladada a la unidad de cuidados intensivos por un cuadro de shock hipovolémico.

Una vez superada esta complicación, se realiza el respectivo seguimiento con las determinaciones seriadas de los niveles de BHCG (fracción beta de gonadotrofina coriónica humana) y estudios complementarios de imagen.

Los niveles de BHCG (fracción beta de gonadotrofina coriónica humana) continuaron elevados en tres determinaciones consecutivas. En el examen ecográfico de control todavía se evidenciaban varias imágenes sugestivas de enfermedad trofoblástica, por esta razón se realizaron nuevas AMEU (aspiración manual endouterina) por dos ocasiones más.

En base a los resultados obtenidos en el respectivo seguimiento y a pesar de no contar con el resultado del examen histopatológico, se decide iniciar un tratamiento quimioterapéutico, según el esquema propuesto por ${ }^{6}$ en base a metotrexato (días 1-3-57). Los controles posteriores de estadía hospitalaria, demostraron que con este esquema terapéutico que los niveles de BCHG (fracción beta de gonadotrofina coriónica humana) disminuyeron de forma considerable. En definitiva, en las cuatro semanas de permanencia en esta de casa de salud se superaron con éxito la mayor parte de las manifestaciones clínicas consideradas al inicio como de gravedad.

El resultado del estudio histopatológico fue de un coriocarcinoma, el cual al asociarse con las demás características de clínicas y de laboratorio, se llegó a la conclusión de que se trataba de una neoplasia trofoblástica gestacional de bajo riesgo. Por tal razón, se decidió su traslado a una unidad de tercer nivel para complementar con los demás estudios necesarios para este caso y su tratamiento. 
Revista Arbitrada Interdisciplinaria de Ciencias de la Salud. SALUD Y VIDA

Volumen 4. Número 8. Año 4. Julio - Diciembre 2020

Hecho el depósito de Ley: FA2016000010

ISSN: 2610-8038

FUNDACIÓN KOINONIA (F.K)

Santa Ana de Coro, Venezuela.

Pedro Ángel Sangucho-Verdezoto; Ana Abigail López-Guerrero; María Margarita Escobar-Martínez; Jenny Irene Plasencia-Criollo

\section{DISCUSIÓN}

Los casos de esta morbilidad en su mayor parte son de tipo benigno, se habla entonces de una mola parcial y una mola completa ${ }^{7}$. Sin embargo, en ocasiones la misma se puede presentar de forma maligna, en este caso se trata de una mola invasiva o de la neoplasia trofoblástica gestacional, con sus diferentes variedades histopatológicas y su capacidad de producir metástasis a estructuras vecinas e inclusive a distancia, que representan aproximadamente el $0.8 \%$ de los casos ${ }^{8}$.

La presentación clínica es una consideración muy importante que se debe tener en cuenta, sobre todo cuando se presenta un caso en el nivel primario de atención. Se debe tomar en cuenta que en estos centros no se cuenta con todos los métodos auxiliares de diagnóstico que son indispensables en estas circunstancias ${ }^{9}$. El cuadro clínico se caracteriza en la mayor parte de los casos por la presencia de un sangrado vaginal en el primer trimestre de gestación, acompañado de una altura de fondo uterino mayor a la que corresponde para la edad gestacional, la presencia de preeclampsia junto con la respectiva sintomatología vasomotora ${ }^{10}$. Además, existe otro tipo de sintomatología que indica la afectación de otros órganos y sistemas, como es el caso de la presencia de trastornos relacionados con la función tiroidea, anemia, entre otros ${ }^{11}$.

El diagnóstico se lo realiza utilizando los hallazgos encontrados en la anamnesis, un examen físico completo y los resultados de los exámenes de laboratorio e imagen. La solicitud de dichas pruebas complementarias debe estar orientadas de acuerdo a la sospecha diagnóstica a la que se ha llegado ${ }^{12}$. Por consiguiente, resulta indispensable la realización de una biometría hemática, tipificación sanguínea, factor $\mathrm{RH}$, química sanguínea; además de pruebas para determinar la función renal y hepática, los tiempos de la coagulación, los niveles séricos de BHCG (fracción beta de gonadotropina coriónica humana). Si existe sintomatología tiroidea, se debe solicitar hormonas tiroideas; todo lo mencionado hasta aquí a nivel sanguíneo ${ }^{13}$.

La ejecución de una ecografía pélvica y transvaginal también resulta importante, en estas imágenes se pueden observar signos característicos que son compatibles con una 
Revista Arbitrada Interdisciplinaria de Ciencias de la Salud. SALUD Y VIDA

Volumen 4. Número 8. Año 4. Julio - Diciembre 2020

Hecho el depósito de Ley: FA2016000010

ISSN: 2610-8038

FUNDACIÓN KOINONIA (F.K).

Santa Ana de Coro, Venezuela.

Pedro Ángel Sangucho-Verdezoto; Ana Abigail López-Guerrero; María Margarita Escobar-Martínez; Jenny Irene Plasencia-Criollo

enfermedad trofoblástica gestacional ${ }^{14}$, se los debe realizar siempre empezando desde una radiografía estándar de tórax, hasta lo más complejo como una tomografía axial computarizada de diferentes regiones corporales en virtud de la sospecha clínica, como en el caso de que se trate de una patología maligna ${ }^{15}$.

El estudio de histopatología nos proporciona información sobre la variedad histológica benigna o maligna de esta enfermedad y así poder establecer un diagnóstico definitivo. De esta manera se podrá tomar una decisión para un tratamiento específico ${ }^{16}$.

El tratamiento en primera instancia una vez realizado un diagnóstico inicial consiste llevar a cabo una aspiración manual endouterina (AMEU) ${ }^{17}$. El manejo posterior de esta morbilidad se lleva a cabo de acuerdo a los hallazgos, la sintomatología y complicaciones que se puedan presentar con la evolución del cuadro clínico. En este punto es indispensable tener un diagnóstico definitivo con la ayuda del examen histopatológico. La quimioterapia y la radioterapia son de gran utilidad en los casos donde se han determinado la presencia de malignidad, con un éxito terapéutico de alrededor del $90 \%$ 18 .

El seguimiento es otro evento que resulta de gran importancia mediante las determinaciones periódicas de los niveles de BHCG (fracción beta de gonadotrofina coriónica humana) a nivel sanguíneo. También son necesarios los estudios de imagen, sobre todo en los casos donde se haya detectado malignidad, estos procedimientos nos proporcionarán información valiosa sobre la respuesta terapéutica y la evolución favorable o no, de las pacientes que tienen este padecimiento 1920 .

Cabe destacar que su manejo clínico, diagnóstico y terapéutico se lo realiza de una forma adecuada según la literatura médica, una de las más relevantes es la descrita por ${ }^{21}$, según la cual el mecanismo para establecer un abordaje adecuado de esta patología coincide en gran parte con el proceso llevado a cabo en el presente caso. ${ }^{22}$ habla de la importancia y eficacia del uso del metotrexato con agente quimioterapéutico de primera línea en el tratamiento de esta enfermedad, con una buena eficacia que llega a más del $90 \%$. 
Revista Arbitrada Interdisciplinaria de Ciencias de la Salud. SALUD Y VIDA

Volumen 4. Número 8. Año 4. Julio - Diciembre 2020

Hecho el depósito de Ley: FA2016000010

ISSN: 2610-8038

FUNDACIÓN KOINONIA (F.K).

Santa Ana de Coro, Venezuela.

Pedro Ángel Sangucho-Verdezoto; Ana Abigail López-Guerrero; María Margarita Escobar-Martínez; Jenny Irene Plasencia-Criollo

Es indiscutible que este caso tuvo un manejo adecuado desde el punto de vista biológico de esta morbilidad. Sin embargo, el enfoque integral del mismo fue casi nulo, debido a que el mayor porcentaje de profesionales que atendieron a la enferma se enfocaron a la parte biológica del problema, dejando a un lado la parte familiar, social y cultural de la paciente ${ }^{23}$.

El enfoque integral de este caso permitió obtener datos importantes que fueron pasados por alto por otros profesionales, entre estos se pueden destacar que la paciente viene de una familia mediana, biparental, bigeneracional, reconstituida, la relación con su madre y hermanos no es buena desde que su progenitora volvió a casarse, la joven pasa por varias crisis familiares de pérdida, de cambio de status. Para ella no existen redes de apoyo, las cuales resultan indispensables que existan para que le ayuden a enfrentar los acontecimientos que vendrán a futuro como causa de su enfermedad 242526 .

\section{Un enfoque médico integral}

La necesidad de un manejo médico integral y multidisciplinario resulta indispensable debido a que la visión actual de la medicina ha dado importantes cambios. Sin embargo, en una buena parte de los casos, no se observa que esto suceda en los diferentes niveles de atención de salud de nuestro país, los profesionales en su mayor parte se limitan a investigar sólo lo biológico, dejando de lado al contexto referente a lo social, cultural y familiar de cada individuo ${ }^{27}$.

El conocimiento de datos que se relacionan con la tipología familiar, el ciclo vital individual y de la familia, las crisis familiares en sus diversos tipos, la funcionalidad familiar; son cosas pasadas por alto la mayor parte de las veces por los médicos tratantes que laboran principalmente en las instituciones hospitalarias ${ }^{28} 29$.

El enfoque integral sin lugar a dudas lo debe llevar a cabo el Médico Familiar, de esta manera, el mismo obtendrá más herramientas para el manejo adecuado de una determinada situación independientemente de su nivel de complejidad. En la visión integral todo lo que se pueda ejecutar debe ir en beneficio del paciente ${ }^{30}$. 
Pedro Ángel Sangucho-Verdezoto; Ana Abigail López-Guerrero; María Margarita Escobar-Martínez; Jenny Irene Plasencia-Criollo

\section{CONCLUSIONES}

El enfoque integral siempre debe ir de la mano de la parte biológica y clínica de una persona con cierto tipo de morbilidad, la asociación de estos aspectos le permitirá al profesional tomar decisiones adecuadas que vayan en beneficio no sólo del paciente, sino de todo su entorno Familiar.

La parte referente a los problemas clínicos y biológicos fueron manejados de la mejor manera posible. Pero resulta indispensable agregarle a este caso la esencia del Médico de Familia, a través de un seguimiento periódico con visitas domiciliarias y la utilización de una serie de instrumentos que se tiene como profesional en esta área, para en conjunto con la paciente y su familia priorizar cada uno de los problemas identificados. Se debe considerar que la información proporcionada sobre la importancia de los controles periódicos completos, se la debe realizar de la manera más clara posible, ya que esto nos ayudará a una identificación temprana cualquier factor de riesgo que puede representar algún peligro para su salud.

\section{CONFLICTO DE INTERÉS}

Los autores declaran que no tienen conflicto de interés en la publicación de este artículo.

\section{FINANCIAMIENTO}

Autofinanciado

\section{AGRADECIMIENTO}

A la Universidad Técnica de Ambato; por motivar el desarrollo la investigación. 
Revista Arbitrada Interdisciplinaria de Ciencias de la Salud. SALUD Y VIDA

Volumen 4. Número 8. Año 4. Julio - Diciembre 2020

Hecho el depósito de Ley: FA2016000010

ISSN: $2610-8038$

FUNDACIÓN KOINONIA (F.K)

Santa Ana de Coro, Venezuela.

Pedro Ángel Sangucho-Verdezoto; Ana Abigail López-Guerrero; María Margarita Escobar-Martínez; Jenny Irene Plasencia-Criollo

\section{REFERENCIAS}

1. Seckl MJ, Sebire NJ, Berkowitz RS. Gestational trophoblastic disease. Lancet. 2010 Aug 28;376 (9742):717-29. doi: 10.1016/S0140-6736(10)60280-2. Epub 2010 Jul 29. PMID: 20673583.

2. Lima LLA, Padron L, Câmara R, Sun SY, Rezende J Filho, Braga A. The role of surgery in the management of women with gestational trophoblastic disease. Rev Col Bras Cir. 2017 Jan-Feb;44(1):94-101. English, Portuguese. doi: 10.1590/010069912017001009. PMID: 28489216.

3. López CL, Lopes VGS, Resende FR, Steim JL, Padrón L, Sun SY, Araujo Júnior E, Braga A. Gestational Trophoblastic Neoplasia after Ectopic Molar Pregnancy: Clinical, Diagnostic, and Therapeutic Aspects. Rev Bras Ginecol Obstet. 2018 May;40(5):294-299. English. doi: 10.1055/s-0038-1653976. Epub 2018 May 16. PMID: 29768638.

4. Berkowitz RS, Goldstein DP. Clinical practice. Molar pregnancy. N Engl J Med. 2009 Apr 16;360(16):1639-45. doi: 10.1056/NEJMcp0900696. PMID: 19369669.

5. Andrijono $A$, Muhilal $M$. Prevention of post-mole malignant trophoblastic disease with vitamin A. Asian Pac J Cancer Prev. 2010;11(2):567-70. PMID: 20843153.

6. van Trommel NE, Massuger LF, Schijf CP, ten Kate-Booij MJ, Sweep FC, Thomas $\mathrm{CM}$. Early identification of resistance to first-line single-agent methotrexate in patients with persistent trophoblastic disease. J Clin Oncol. 2006 Jan 1;24(1):528. doi: 10.1200/JCO.2005.03.3043. PMID: 16382113.

7. van Trommel NE, Thomas CM, Massuger LF, Sweep FC. Second curettage in persistent trophoblastic disease (PTD): the need for univocal definition of PTD. Gynecol Oncol. 2005 Oct;99(1):250-1; author reply 251. doi: 10.1016/j.ygyno.2005.03.012. PMID: 16224821.

8. Pezeshki M, Hancock BW, Silcocks P, Everard JE, Coleman J, Gillespie AM, Tidy $\mathrm{J}$, Coleman RE. The role of repeat uterine evacuation in the management of persistent gestational trophoblastic disease. Gynecol Oncol. 2004 Dec;95(3):4239. doi: 10.1016/j.ygyno.2004.08.045. PMID: 15581942. 
Revista Arbitrada Interdisciplinaria de Ciencias de la Salud. SALUD Y VIDA

Volumen 4. Número 8. Año 4. Julio - Diciembre 2020

Hecho el depósito de Ley: FA2016000010

ISSN: 2610-8038

FUNDACIÓN KOINONIA (F.K).

Santa Ana de Coro, Venezuela.

Pedro Ángel Sangucho-Verdezoto; Ana Abigail López-Guerrero; María Margarita Escobar-Martínez; Jenny Irene Plasencia-Criollo

9. Maestá I, Braga A. Desafios do tratamento de pacientes com doença trofoblástica gestacional [Challenges of the treatment of patients with gestational trophoblastic disease]. Rev Bras Ginecol Obstet. 2012 Apr;34(4):143-6. Portuguese. PMID: 22584808.

10. Tidy JA, Gillespie AM, Bright N, Radstone CR, Coleman RE, Hancock BW. Gestational trophoblastic disease: a study of mode of evacuation and subsequent need for treatment with chemotherapy. Gynecol Oncol. 2000 Sep;78(3 Pt 1):30912. doi: 10.1006/gyno.2000.5839. PMID: 10985885.

11. Mangili G, Lorusso D, Brown J, Pfisterer J, Massuger L, Vaughan M, Ngan HY, Golfier F, Sekharan PK, Charry RC, Poveda A, Kim JW, Xiang Y, Berkowtiz R, Seckl MJ. Trophoblastic disease review for diagnosis and management: a joint report from the International Society for the Study of Trophoblastic Disease, European Organisation for the Treatment of Trophoblastic Disease, and the Gynecologic Cancer InterGroup. Int J Gynecol Cancer. 2014 Nov;24(9 Suppl 3):S109-16. doi: 10.1097/IGC.0000000000000294. PMID: 25341573.

12. Seckl MJ, Sebire NJ, Fisher RA, Golfier F, Massuger L, Sessa C; ESMO Guidelines Working Group. Gestational trophoblastic disease: ESMO Clinical Practice Guidelines for diagnosis, treatment and follow-up. Ann Oncol. 2013 Oct;24 Suppl 6:vi39-50. doi: 10.1093/annonc/mdt345. Epub 2013 Sep 1. PMID: 23999759.

13. Bolze PA, Attia J, Massardier J, Seckl MJ, Massuger L, van Trommel N, Niemann I, Hajri T, Schott AM, Golfier F; EOTTD group. Formalised consensus of the European Organisation for Treatment of Trophoblastic Diseases on management of gestational trophoblastic diseases. Eur J Cancer. 2015 Sep;51(13):1725-31. doi: 10.1016/j.ejca.2015.05.026. Epub 2015 Jun 16. PMID: 26092638.

14. Rivera Domínguez A, Mora Jurado A, García de la Oliva A, de Araujo MartinsRomeo D, Cueto Álvarez L. Gynecological pelvic pain as emergency pathology. Radiologia. $2017 \quad$ Mar-Apr;59(2):115-127. English, Spanish. doi: 10.1016/j.rx.2016.09.010. Epub 2016 Dec 12. PMID: 27979433.

15. Ngan HYS, Seckl MJ, Berkowitz RS, Xiang Y, Golfier F, Sekharan PK, Lurain JR, Massuger $\mathrm{L}$. Update on the diagnosis and management of gestational trophoblastic disease. Int J Gynaecol Obstet. 2018 Oct;143 Suppl 2:79-85. doi: 10.1002/ijgo.12615. PMID: 30306586. 
Revista Arbitrada Interdisciplinaria de Ciencias de la Salud. SALUD Y VIDA

Volumen 4. Número 8. Año 4. Julio - Diciembre 2020

Hecho el depósito de Ley: FA2016000010

ISSN: 2610-8038

FUNDACIÓN KOINONIA (F.K).

Santa Ana de Coro, Venezuela.

Pedro Ángel Sangucho-Verdezoto; Ana Abigail López-Guerrero; María Margarita Escobar-Martínez; Jenny Irene Plasencia-Criollo

16. Espinoza AA, Fernández VR, Solar VT. Update in gestational trophoblastic pathology: hydatidiform mole and neoplasias. Revista Médica Sinergia. 2019;4(05):44-59.

17. Ning $F$, Hou $H$, Morse AN, Lash GE. Understanding and management of gestational trophoblastic disease. F1000Res. 2019 Apr 10;8:F1000 Faculty Rev428. doi: 10.12688/f1000research.14953.1. PMID: 31001418; PMCID: PMC6464061.

18. Melamed A, Gockley AA, Joseph NT, Sun SY, Clapp MA, Goldstein DP, Berkowitz RS, Horowitz NS. Effect of race/ethnicity on risk of complete and partial molar pregnancy after adjustment for age. Gynecol Oncol. 2016 Oct;143(1):73-76. doi: 10.1016/..ygyno.2016.07.117. Epub 2016 Jul 30. PMID: 27486130.

19. Shaaban AM, Rezvani M, Haroun RR, Kennedy AM, Elsayes KM, Olpin JD, Salama ME, Foster BR, Menias CO. Gestational Trophoblastic Disease: Clinical and Imaging Features. Radiographics. 2017 Mar-Apr;37(2):681-700. doi: 10.1148/rg.2017160140. PMID: 28287945.

20. Kaur B, Short D, Fisher RA, Savage PM, Seckl MJ, Sebire NJ. Atypical placental site nodule (APSN) and association with malignant gestational trophoblastic disease; a clinicopathologic study of 21 cases. Int J Gynecol Pathol. 2015 Mar;34(2):152-8. doi: 10.1097/PGP.0000000000000128. PMID: 25675185.

21. Mangili G, Sigismondi C, Lorusso D, Cormio G, Candiani M, Scarfone G, Mascilini F, Gadducci A, Mosconi AM, Scollo P, Cassani C, Pignata S, Ferrandina G. The role of staging and adjuvant chemotherapy in stage I malignant ovarian germ cell tumors (MOGTs): the MITO-9 study. Ann Oncol. 2017 Feb 1;28(2):333-338. doi: 10.1093/annonc/mdw563. PMID: 27803008.

22. Frijstein MM, Lok CAR, van Trommel NE, Ten Kate-Booij MJ, Massuger LFAG, van Werkhoven E, Kaur B, Tidy JA, Sarwar N, Golfier F, Winter MC, Hancock BW, Seckl MJ; all the contributors to the ISSTD PSTT/ETT database. Management and prognostic factors of epithelioid trophoblastic tumors: Results from the International Society for the Study of Trophoblastic Diseases database. Gynecol Oncol. 2019 Feb;152(2):361-367. doi: 10.1016/j.ygyno.2018.11.015. Epub 2018 Nov 22. PMID: 30473257.

23. Irigoyen-Coria A, Vázquez-Martínez VH, Arévalo-Ramírez LA. Influencia de los médicos de Tamaulipas en el desarrollo de la Medicina Familiar en México y Latinoamérica. Aten Fam. 2016;23(1):1-3. 
Pedro Ángel Sangucho-Verdezoto; Ana Abigail López-Guerrero; María Margarita Escobar-Martínez; Jenny Irene Plasencia-Criollo

24. Martín Zurro A y cols. Compendio de Atención primaria. Conceptos, organización y práctica clínica en Medicina de Familia. 4th Ed. Barcelona: Elsevier; 2016.

25. Varela-Rueda CE, Reyes-Morales $\mathrm{H}$, Albavera-Hernández $\mathrm{C}$, et al. La medicina familiar en México: presente y futuro. Gac Med Mex. 2016;152(1):135-140.

26. De la Cruz-Florencio P, Godínez-Tamay ED, Hernández-Miranda MB. La Medicina Familiar como especialidad médica: percepción de otros especialistas. Aten Fam. 2016;23(3):109-112.

27. Sánchez Hidalgo Md. La familia como objeto de investigación: un reto para los profesionales de la salud. RM [revista en Internet]. 2016; 20(5): [aprox. 4 p.].

28. Sandoval M, Holme F, Lobo S, Slavkovsky R, Thomson KA, Jeronimo J, Figueroa $\mathrm{J}$, Sanjose S. Age patterns of human papillomavirus infection as primary screening test for cervical cancer and subsequent triage with visual inspection in Honduras. Salud Publica Mex. 2020 Sep-Oct;62(5):487-493. English. doi: 10.21149/10979. Epub 2020 Jul 15. PMID: 32697901.

29. Flores-Pulido JJ, Martínez-Correa M. Cáncer cervicouterino y virus del papiloma humano. Una mirada desde el enfoque médico familiar [Cervical cancer and human papillomavirus. A glance from a family medical viewpoint]. Rev Med Inst Mex Seguro Soc. 2015;53 Suppl 2:S162-4. Spanish. PMID: 26462511.

30. Pérez Pérez Alvaro, Gonzáles Moreno Ivian Zoila, Rodríguez Larralde Raúl Lázaro, Terrero Quiala Lisbet. Agregación familiar para cáncer de cuello uterino. Rev Cubana Med Gen Integr [Internet]. 2019 Dic ; 35(4): e881. 